\title{
Visibilidad y producción científica de las Tesis Doctorales de Historia del Periodismo en las Universidades Españolas (2002-2012)
}

\author{
Carlos OLIVA MARAÑóN \\ Universidad Rey Juan Carlos \\ carlos.oliva.maranon@urjc.es
}

\begin{abstract}
Resumen:
Las Tesis Doctorales constituyen una tipología documental muy relevante para evaluar el conocimiento. El objetivo de esta investigación es indagar la producción científica doctoral en Historia del Periodismo en el período 2002-2012. La muestra está avalada por 93 Tesis Doctorales a través de las variables de frecuencia, año de defensa, Departamento y Universidad. Los resultados constatan la primacía de Cataluña como el ámbito investigador más fructífero en esta área; el predominio del Departamento de Comunicación; y la prevalencia del año 2009 como el de mayor producción doctoral.
\end{abstract}

Palabras clave: Tesis Doctorales; Historia del Periodismo; evaluación del conocimiento; Espacio Europeo de Educación Superior (EEES); Universidades Españolas.

\section{Visibility and scientific production of Doctoral Theses History of Journalism in the Spanish Universities}

(2002-2012)

\begin{abstract}
Abstrac:
The Doctoral Thesis constitute a very relevant document types to assess knowledge. The objective of this research is to investigate doctoral scientific production in History of Journalism in the period 20022012. The sample is supported by $93 \mathrm{PhD}$ Thesis by frequency variables, year of defense, Department and University. The results indicate the primacy of Catalonia as the most fruitful field of research in this area; the predominance of the Department of Communication, and the prevalence of 2009 as the most productive doctoral.
\end{abstract}

Key Words: Doctoral Thesis; History of Journalism; assessment of knowledge; European Higher Education Area (EHEA); Spanish Universities.

Referencia normalizada:

Oliva Marañon, C. (2014): Visibilidad y producción científica de las tesis doctorales de Historia del Periodismo en las universidades españolas (2002-2012). Historia y Comunicación Social. Vol. 19. Núm. Especial Febrero. Págs. 183-193.

Sumario: 1. Introducción. 2. Metodología. 3. Resultados 4. Discusión de resultados. 5. Conclusiones. 6. Referencias bibliográficas. 


\section{Introducción}

Desde que en 1935, coincidiendo con los primeros trabajos de Bradford, Ortega y Gasset hiciera una primera referencia en España al análisis cuantitativo de la Documentación, la Bibliometría ha tenido un discreto desarrollo en nuestro país. Alan Pritchard fue el primero que definió el término "Bibliometría" como "la aplicación de los métodos estadísticos y matemáticos dispuestos para definir los procesos de la comunicación escrita y la naturaleza y el desarrollo de las disciplinas científicas mediante técnicas de recuento y análisis de dicha comunicación" (Pritchard, 1969: 348).

Además, se manifiesta un gran interés de la Bibliometría en "los estudios de Tercer Ciclo como marco adecuado para la consecución y transmisión de los avances científicos y como herramienta para la formación de investigadores que puedan afrontar con éxito el reto que suponen las nuevas metodologías, ciencias y técnicas de la sociedad actual" (Valcárcel, 2002: 13).

Entre 1971 y 1973 se publican los primeros trabajos, cuyos autores son María Luz Terrada y José María López Piñero. En 1972, Piñero publica el primer libro dedicado al tema: El análisis estadístico y sociométrico de la literatura científica. Al año siguiente, el mismo autor traduce el libro Price Little Science, Big Science. En esta década se publican varios artículos con un enfoque bibliométrico. Autores como los mencionados López Piñero (1972), María Luz Terrada (1973, 1980 y 1981), José Ramón Pérez Álvarez-Ossorio (1980 y 1988), Heliodoro Carpintero (1981) y otros son pioneros en la utilización de esta metodología.

En los años setenta se configuran tres grupos principales de investigación: el primero, en torno a López Piñero y María Luz Terrada, pertenecientes a la Facultad de Medicina de Valencia y al Instituto de Estudios Documentales e Históricos sobre la Ciencia (CSIC), trabaja en el campo de la Medicina. El segundo, fundado por el psicólogo Heliodoro Carpintero, encabeza un grupo de investigación en Psicología con autores como José María Peiró, Francisco Tortosa, Enrique Carbonell o Luis Montoro, entre otros. Por último, también a mediados de los setenta, surge en Madrid otro grupo en el Instituto de Información y Documentación en Ciencia y Tecnología (ICYT), perteneciente al Consejo Superior de Investigaciones Científicas (CSIC). Este grupo realiza estudios bibliométricos en el área de Ciencia; particularmente la Química. Se inicia con investigadores como Pérez Álvarez-Ossorio, ampliándose a lo largo de los ochenta con investigadores del propio ICYT (Luis Ferreiro, Isabel Gómez Caridad, Rosa Ancho, Aida Méndez, Adelaida Román, Rosa de la Viesca, Godofredo López, Carmen Galbán, Manuela Vázquez, etc.).

Posteriormente, el Informe de Miguel Valcárcel Cases (2002), Catedrático de la Universidad de Córdoba, propone los siguientes factores que hay que tener en cuenta para lograr una calidad integral de las Tesis Doctorales:

- El aumento de medios humanos y materiales, el reconocimiento de la labor docente del profesorado y de las tareas de dirección. 
- La fijación de criterios para evaluar los Programas de Doctorado.

- La responsabilidad de los Departamentos en asegurar unos mínimos de calidad de las Tesis Doctorales antes de pasar a la fase de presentación y defensa.

- La mejora de los sistemas de evaluación.

- El establecimiento de indicadores de evaluación de los estudios de Doctorado.

Además, algunos autores han partido de la naturaleza de las Tesis como literatura gris y su importancia para los investigadores. Es el caso de Moreno-Torres (1986: 671-696), López López (1996: 84-89), M. R. Moralejo (2000: 236-243) y Aurora de Miguel (2000: 431-437). Otros como Merlo y Sorli (2002: 95-103) y Luisa Orera (2004: 91-102) han incidido en la búsqueda del control bibliográfico y del acceso físico a las investigaciones doctorales.

En el ámbito de la calidad, uno de los objetivos específicos postula "ofrecer herramientas específicas para la mejora de la calidad del Doctorado, tales como la recogida sistemática de información cuantitativa y cualitativa, la definición de indicadores y estándares de calidad, el diseño de sistemas de autorregulación y acreditación, entre otras" (Valcárcel, 2000: 16) y porque, efectivamente, una adecuada evaluación de las Tesis Doctorales se traduce en uno de los factores inherentes a su calidad.

En lo referente al periodismo, una visión integral de su historia plantea problemas de interpretación en cuanto a la evolución de cada país y sus factores específicos de progreso y atraso, que sólo pueden resolverse con el auxilio de la historia comparada. De otra forma sería muy difícil ofrecer una explicación clara y razonada de las acusadas diferencias que presentan las principales tradiciones nacionales, no solamente entre Europa y América, sino en el mismo viejo continente, incluso antes de que empiece a desarrollarse la prensa en Estados Unidos (Guillamet, 2003: 36).

En este sentido, una visión integral de la Historia del Periodismo sugiere una asociación tácita de sus grandes líneas de evolución con un grupo reducido de países de referencia -Italia, Alemania, Inglaterra, Francia, España y Estados Unidos, en relación con sus sucesivas aportaciones e influencias para su desarrollo y expansión-, frente a un segundo gran grupo que engloba al resto de países desarrollados y a un tercero y más amplio, relativo a los países en vías de desarrollo (Guillamet, 2003: 37).

Igualmente, en opinión de Pascal Lardelier (1999: 7), Armand Mattelart afirmaba a principios de los años 90 que "el olvido de la Historia es uno de los rasgos recurrentes del pensamiento sobre la comunicación". Efectivamente, gran parte de los investigadores y teóricos en Ciencias de la Información y de la Comunicación se han ocupado, hasta fechas muy recientes, en objetos que han considerado más "modernos" o "contemporáneos", y que tuvieran la característica de la semejanza con su disciplina. Desde los años cincuenta, se ha tomado conciencia de la exacta función y amplitud del fenómeno informativo; se ha pasado de las "historias de periódicos" a la de la comunicación como sistema o conjunto compuesto por un determinado número de elementos, se ha saltado de la mera enumeración de títulos y leyes a la utilización 
de modelos formales. Además, Lardelier (1999: 21-22) sostiene que Philippe Breton aseveraba: "hablar de la Historia de la Comunicación implica, naturalmente, que uno defina con precisión lo que se entiende por Comunicación".

Por su parte, Ramón Sala (2007: 13) reflexiona acerca del concepto de Historia de los Medios de Comunicación de Masas y afirma:

- Es una historia que tiene un campo "específico".

- Los medios de comunicación tienen su historia, pero son también documentos para construir la Historia. Esto es necesario subrayarlo, porque no se tiene suficientemente en cuenta.

- El estudio de la evolución de estos medios puede constituir una especie de ciencia auxiliar (apoyar a la Historia general).

Por lo que respecta al desarrollo y evolución de la Comunicación Social en general, y la Historia del Periodismo en particular, es una de las materias que más interés ha despertado entre los investigadores del campo de la Comunicación. En los planes de estudio de Grado, adaptados al Espacio Europeo de Educación Superior (EEES), es una asignatura de Formación Básica (FB). Esta circunstancia ha motivado que dicha materia sea analizada no sólo desde el plano de la investigación, sino también desde el plano docente. La impartición de asignaturas tales como "Teoría e Historia del Periodismo", "Historia del Periodismo Español", "Historia del Periodismo Universal", "Desarrollo de la Comunicación Social" o "Historia de la Comunicación Social", entre otras, viene acompañada de una considerable publicación científica (Haro y Martínez 2011: 224).

\section{Metodología}

Los objetivos de esta investigación son los siguientes:

- Investigar la producción científica en el área de Historia del Periodismo mediante las Tesis Doctorales defendidas en el período 2002-2012 en las Universidades Españolas.

- Conocer el año de su defensa pública.

- Averiguar en qué Universidad se han presentado.

- Saber qué Departamento ha sido el responsable de su inscripción y presentación.

La metodología empleada se ha basado en la utilización de TESEO, Base de Datos del Ministerio de Educación y Ciencia en la que se recogen las Tesis Doctorales defendidas en las Universidades Españolas desde 1976. Se ha considerado pertinente investigar la producción científica en el área de Historia del Periodismo mediante las Tesis Doctorales defendidas en el período 2002-2012 en las Universidades Españo- 
las. Para ello, se ha tomado como referencia este ámbito temporal, ya que se encuentran consolidadas las Facultades y los Departamentos de Ciencias de la Comunicación, los estudios de Periodismo, así como el Tercer Ciclo. Por tanto, estas tipologías documentales, denominadas también "literatura gris", se configuran como una de las Fuentes de Información más pertinentes para estudiar el estado de la investigación en un país a través de su literatura científica.

Además, la Base de Datos TESEO es referencial y cada registro contiene los siguientes campos informativos:

- Clave.

- Autor.

- Título de la Tesis.

- Director de la Tesis.

- Universidad.

- Facultad.

- Tribunal.

- Calificación obtenida.

- Centro de trabajo.

- Curso académico.

- Descriptores de materias.

- Descriptores UNESCO.

- Resumen de la Tesis.

Fundamentalmente, se ha realizado la siguiente estrategia documental de búsqueda:

- En primer lugar, en el campo de "curso académico", se ha seleccionado desde 2002-2003 hasta 2011-2012.

- En segundo lugar, en el campo de "palabras clave" y "materia", y dentro del Tesauro de Materias UNESCO, se ha elegido "Historia del Periodismo" (Código: 550611). Los resultados son 93 Tesis Doctorales.

- En tercer lugar, en el campo de "mostrar resultados", se ha seleccionado la opción de 50 resultados por página, máxima que permite la interfaz de la base de datos.

Una vez recopiladas las Tesis Doctorales pertinentes para la investigación, se ha realizado un análisis de la información mediante tres variables:

- Año de publicación. Su finalidad es comprobar el volumen de producción científica durante los años investigados. Los años de las Tesis Doctorales suelen 
diferir de unas Bases de Datos a otras. Por ello, para establecer unos criterios comunes, se ha tomado el año registrado en la Base de Datos TESEO.

- Universidad. Su objetivo es conocer en qué Universidades se ha realizado la defensa de las Tesis Doctorales objeto de esta investigación.

- Departamento. Mediante esta variable se demuestra si todas las Tesis Doctorales se han realizado en Departamentos de Comunicación o en otros Departamentos de diferentes áreas de conocimiento.

\section{Resultados}

Los resultados se han categorizado por volumen de producción científica por años, Universidades en las que se han defendido las Tesis Doctorales y Departamentos universitarios responsables de su presentación.

Volumen de producción científica por años (tabla I)

(Campo de palabra clave y materia UNESCO: 550611 Historia del Periodismo)

\begin{tabular}{|c|c|c|}
\hline AÑOS & FRECUENCIA & PORCENTAJE \\
\hline 2004 & 2 & $2,15 \%$ \\
\hline 2005 & 11 & $11,83 \%$ \\
\hline 2006 & 12 & $12,90 \%$ \\
\hline 2007 & 7 & $7,53 \%$ \\
\hline 2008 & 10 & $10,75 \%$ \\
\hline 2009 & 15 & $16,12 \%$ \\
\hline 2010 & 14 & $15,06 \%$ \\
\hline 2011 & 14 & $15,06 \%$ \\
\hline 2012 & 8 & $8,60 \%$ \\
\hline \hline TOTAL & 93 & $100,00 \%$ \\
\hline
\end{tabular}

Fuente: elaboración propia

Tras el análisis de la producción científica por años, se pueden extraer las siguientes premisas:

- La producción científica alcanza su cota máxima en 2009, con 15 Tesis Doctorales $(16,12 \%)$; seguido de 2010 y 2011, con 14 (15,06\%); 2006, con 12 $(12,90 \%)$; y 2005 , con $11(11,83 \%)$.

- $\quad$ Sin embargo, los años con menor producción científica son 2007, con 7 Tesis Doctorales $(7,53 \%)$; y 2004, con $2(2,15 \%)$. 
Universidades en las que se han defendido las Tesis Doctorales (tabla II)

\begin{tabular}{|l|c|c|}
\hline \multicolumn{1}{|c|}{ UNIVERSIDAD } & FRECUENCIA & PORCENTAJE \\
\hline Universidad Autónoma de Barcelona & 9 & $9,67 \%$ \\
\hline Universidad de Sevilla & 9 & $9,67 \%$ \\
\hline Universidad de La Laguna & 8 & $8,60 \%$ \\
\hline Universidad de Valladolid & 5 & $5,38 \%$ \\
\hline Universidad Complutense de Madrid & 4 & $4,30 \%$ \\
\hline Universidad de Navarra & 4 & $4,30 \%$ \\
\hline Universidad de Barcelona & 4 & $4,30 \%$ \\
\hline Universidad de Salamanca & 3 & $3,23 \%$ \\
\hline Universidad Pontificia de Salamanca & 3 & $3,23 \%$ \\
\hline Universidad de Málaga & 3 & $3,23 \%$ \\
\hline Universidad de Girona & 3 & $3,23 \%$ \\
\hline Universidad de Santiago & 3 & $3,23 \%$ \\
\hline Universidad de Valencia & 3 & $3,23 \%$ \\
\hline Universidad CEU San Pablo & 3 & $3,23 \%$ \\
\hline Universidad Pompeu Fabra & 3 & $3,23 \%$ \\
\hline Universidad de Zaragoza & 2 & $2,16 \%$ \\
\hline Universidad de Granada & 2 & $2,16 \%$ \\
\hline Universidad de Alcalá & 2 & $2,16 \%$ \\
\hline Universidad de Cádiz & 2 & $2,16 \%$ \\
\hline Universidad Jaume I & 2 & $2,16 \%$ \\
\hline Universidad de La Coruña & 2 & $2,16 \%$ \\
\hline Universidad Internacional de Cataluña & 1 & $1,07 \%$ \\
\hline Universidad de Huelva & 1 & $1,07 \%$ \\
\hline Universidad de Murcia & 1 & $1,07 \%$ \\
\hline Universidad Pablo de Olavide & 1 & $1,07 \%$ \\
\hline Universidad Politécnica de Valencia & $1,07 \%$ \\
\hline Universidad de La Rioja & 1 & $1,07 \%$ \\
\hline Universidad Autónoma de Madrid & 1 & $1,07 \%$ \\
\hline Universidad de Alicante & $1,07 \%$ \\
\hline Universidad Carlos III de Madrid & 1 & $1,07 \%$ \\
\hline Universidad Ramón Llull & $1,07 \%$ \\
\hline Universidad de Almería & $1,07 \%$ \\
\hline Universidad CEU Cardenal Herrera & $1,07 \%$ \\
\hline Universidad del País Vasco & $1,07 \%$ \\
\hline
\end{tabular}




\begin{tabular}{||l|c|c|}
\hline Universidad de Oviedo & 1 & $1,07 \%$ \\
\hline TOTAL & 93 & $100,00 \%$ \\
\hline
\end{tabular}

Fuente: elaboración propia

Con la finalidad de averiguar en qué Universidades españolas se han realizado estas Tesis Doctorales, se ha procedido a agruparlas por Universidad. Los resultados han sido los siguientes:

- El 22,57\% del total de Tesis defendidas se han realizado en Universidades ubicadas en Cataluña: un 9,67\% en la Universidad Autónoma de Barcelona; un 4,30\% en la Universidad de Barcelona; un 3,23\% en la Universidad de Girona y en la Universidad Pompeu Fabra; y un $1,07 \%$ en la Universidad Internacional de Cataluña y en la Universidad Ramón Llull.

- Un 20,43\% se han realizado en las Universidades de la Comunidad Autónoma de Andalucía: un 9,67\% en la Universidad de Sevilla; un 3,23\% en la Universidad de Málaga; un 2,16\% en la Universidades de Granada y Cádiz; y el 1,07\% en las Universidades de Huelva, Pablo de Olavide y Almería.

- Otro 11,84\% han sido elaboradas en las Universidades de la Comunidad Autónoma de Castilla y León: un 5,38\% en la Universidad de Valladolid; y un $3,23 \%$ en la Universidad de Salamanca y en la Universidad Pontificia de Salamanca.

- El 11,82\% han sido realizadas en las Universidades de la Comunidad Autónoma de Madrid: un 4,3\% en la Universidad Complutense de Madrid; un $3,23 \%$ en la Universidad CEU San Pablo; un 2,15\% en la Universidad de Alcalá; y un 1,07\% tanto en la Universidad Autónoma de Madrid como en la Universidad Carlos III.

- Un 8,6\% han sido elaboradas en Canarias, a través de la Universidad de La Laguna.

- El 8,6\% han sido defendidas en la Comunidad Valenciana, concretamente, el $3,23 \%$ en la Universidad de Valencia; el 2,16\% en la Universidad Jaume I; y el $1,07 \%$ en las Universidades CEU Cardenal Herrera, Alicante y Politécnica de Valencia.

- Otro 5,39\% se han defendido en las Universidades de la Comunidad Autónoma de Galicia: un 3,23\% en la Universidad de Santiago y un 2,16\% en la Universidad de La Coruña.

- Un 4,3\% se han realizado en la Universidad de Navarra.

- Otro 1,07\% han sido realizadas en las Universidades de La Rioja, País Vasco, Oviedo y Murcia. 
Departamentos en los que se han defendido las Tesis Doctorales (tabla III)

\begin{tabular}{|l|c|c|}
\hline \multicolumn{1}{|c|}{ DEPARTAMENTOS } & FRECUENCIA & PORCENTAJE \\
\hline Comunicación & 53 & $56,99 \%$ \\
\hline Historia & 23 & $24,73 \%$ \\
\hline Filología & 17 & $18,28 \%$ \\
\hline TOTAL & 93 & $100,00 \%$ \\
\hline
\end{tabular}

Fuente: elaboración propia

Por lo que respecta a los Departamentos de las Universidades en los que se han defendido las Tesis Doctorales, se pueden extrapolar los siguientes datos:

- El Departamento de Comunicación es en el que se han presentado más Tesis Doctorales (53), lo que supone un 56,99\%. Le siguen, en orden de importancia, los Departamentos de Historia, con 23 (24,73\%); y de Filología, con 17 $(18,28 \%)$.

\section{Discusión de resultados}

En cuanto a la producción científica, el número de Tesis Doctorales defendidas en el área de Historia del Periodismo asciende a 93, siendo el período 2007-2012 el de mayor número, con 68 (73,11\%). Además, en el año 2009 se defendieron 15 Tesis Doctorales en este ámbito.Por tanto, se observa un incremento de la investigación en Historia del Periodismo desde 2002 hasta 2012, cobertura temporal del período investigado. Por lo que respecta a las Universidades en las que se han presentado las Tesis Doctorales, predominan las de Sevilla y Autónoma de Barcelona, con 9; seguida de la Universidad de La Laguna, con 8; y la Universidad de Valladolid, con 5 , lo que evidencia la relevancia de esta área de conocimiento en estos Centros de Enseñanza Superior. Igualmente, las Universidades de Cataluña se sitúan en primer lugar en cuanto al número de Tesis defendidas, ya que ascienden a 21 , lo que supone un $22,58 \%$ del total. En lo referente a los Departamentos, el 56,99\% de las presentadas se han realizado en el de Comunicación.

\section{Conclusiones}

En los últimos años, tras el desarrollo de la Bibliometría, han surgido estudios e investigaciones de diferentes autores acerca de la producción científica mediante el análisis de las Tesis Doctorales en distintas áreas de conocimiento. Igualmente, ha contribuido a ello la consolidación de las Bases de Datos como Fuentes de Información pertinentes para cuantificar la visibilidad de las publicaciones. Estas aplicaciones documentales, fundamentales para la investigación, se caracterizan, por un lado, 
por su gran capacidad de almacenamiento y, por otro, por la estructura y organización de los datos en campos normalizados. Durante el período 2002-2012, se han incrementado las Tesis Doctorales defendidas en las Universidades Españolas en el área de Historia del Periodismo, siendo las de Cataluña las de mayor volumen. En cuanto a los Departamentos responsables de su defensa, predominan los del área de Comunicación, aunque se observa un crecimiento en los de Historia y Filología. Por tanto, las investigaciones acerca de la Historia del Periodismo consolidan a docentes e investigadores como los principales beneficiarios de este conocimiento.

\section{Bibliografía}

CARPINTERO CAPELL, H. (1981). Psicología contemporánea: teoría y métodos cuantitativos para el estudio de su literatura científica. Valencia: Alfaplus.

DELGADO LÓPEZ-CÓZAR, E. (2002). La investigación en Biblioteconomía y Documentación. Gijón: Trea.

GUILLAMET, J. (2003). "Por una historia comparada del periodismo. Factores de progreso y atraso". En: Doxa Comunicación. Revista Interdisciplinar de Estudios de Comunicación y Ciencias Sociales, n. ${ }^{\circ}$ 1, p. 35-56.

HARO DE SAN MATEO, M. a V. de y MARTÍNEZ MÉNDEZ, F. J. (2011). “Análisis temático de los artículos sobre Historia del Periodismo publicados en las revistas científicas españolas de Comunicación”. En: Documentación de las Ciencias de la Información, vol. 34, p. 223-239.

LARDELIER, P. (1999). "Préambule". En: MEI (Médiation et Information), n. ' 10, p. 7-16.

(1999). “Entretiens”. En: MEI (Médiation et Information), n. ${ }^{\circ}$ 10, p. 20-31.

LÓPEZ LÓPEZ, P. (1996). "La investigación bibliométrica en España (Tesis Doctorales)". En: Revista Española de Documentación Científica, vol. 19, n. ${ }^{\text {1, p. }}$ 84-89.

LÓPEZ PIÑERO, J. M. (1972). El análisis estadístico y sociométrico de la literatura científica. Valencia: Centro de Documentación e Informática Médica.

MERLO VEGA, J. A. y SORLI ROJO, A. (2002). "Bases de datos y recursos en Internet sobre Tesis Doctorales". En: Revista Española de Documentación Cientifica, vol. 25, n. ${ }^{\circ} 1$, p. 95-106.

MIGUEL ALONSO, A. D. (2000). “Aportaciones al estudio de la literatura gris universitaria: La evolución de la Tesis Doctoral en España”. En I Congreso Universitario de Ciencias de la Documentación: Teoría, historia y metodología de las Ciencias de la Documentación. Madrid: Universidad Complutense de Madrid, Facultad de Ciencias de la Información, p. 431-437.

MORALEJO ÁLVAREZ, M. ${ }^{a}$ R. (2000). "Las Tesis Doctorales de las Universidades españolas: Control bibliográfico y acceso". En: Revista General de Información y Documentación, vol. 10, n. ${ }^{\circ} 1$, p. 236-243.

MOREIRO GONZÁleZ, J. A. (2000). Manual de Documentación Informativa. Madrid: Cátedra. 
MORENO-TORRES SÁNCHEZ, R. (1986). "El acceso a la literatura gris: Actas de congresos y Tesis Doctorales". En: Boletín de la ANABAD, n. ${ }^{\circ} 4$, p. 671-696.

ORERA ORERA, L. (2004). "Las Tesis sobre Biblioteconomía y bibliotecas y su accesibilidad a través de las bibliotecas universitarias españolas”. En FERNÁNDEZ BAJÓN, M. ${ }^{a}$ T.; LÓPEZ LÓPEZ, P.; LÓPEZ YEPES, J. (Coords.) (2004). Estudios de Biblioteconomía y Documentación. Homenaje a la profesora María Rosa Garrido Arilla. Madrid: Universidad Complutense de Madrid, EUBD, p. 91-102.

PÉREZ-ÁLVAREZ OSSORIO, J. R. (1980). La documentación y la información para el medio ambiente. Panorama general de la información científica y técnica en España y América Latina. Madrid: CIFCA.

PRITCHARD, A. (1969). "Statiscal bibliography or Bibliometrics". En: Journal of Documentation, vol. 25, n. ${ }^{\circ}$, p. 348-369.

SALA NOGUER, R. (2007). Introducción a la Historia de los Medios. Consideraciones teóricas básicas sobre la Historia de los Medios de Comunicación de Masas. Barcelona: Universidad Autónoma de Barcelona.

TERRADA, M. ${ }^{\mathrm{a}}$ L. (1981). Bibliometría de la producción y el consumo de literatura médica en España: 1973-1977. Valencia: Universidad de Valencia, Servicio de Publicaciones.

(1980). Bibliometría de la literatura científica española publicada en revista extranjeras: 1973-1977. Valencia: Universidad de Valencia, Servicio de Publicaciones.

(1973). La literatura médica española contemporánea. Valencia: Centro de Documentación e Informática Médica.

VALCÁRCEL CASES, M. [et al.] (2002). Informe sobre el Doctorado en las Universidades españolas. Situación actual y propuestas de mejora. Disponible en: www.universia.es/contenidos/gestion/Estudio_situación_doctorado_propuestas_mejora.pdf [30-10-2013].

\section{El autor}

Carlos Oliva Marañón. Profesor de la Facultad de Ciencias de la Comunicación de la Universidad Rey Juan Carlos, Departamento de Ciencias de la Comunicación I. Doctor en Ciencias de la Comunicación. Autor, entre otras publicaciones, de las siguientes: Calidad, visibilidad e interactividad: paradigmas de las Universidades Virtuales en España; Las monografias de Publicidad a través de las aplicaciones documentales ISBN y REBIUN: un estudio bibliográfico (2008-2012); El Grado en Cine en las Universidades de España: visibilidad, competencias, contenidos y propuestas de futuro; Creatividad, Publicidad y Educación Emocional: fundamentos del Lipdub como aplicación de la imagen de marca de las Universidades; Fundamentos lingüísticos del lenguaje periodístico y juridico-administrativo. 